\title{
ARCH FILAMENTS ASSOCIATED WITH THE FORMATION OF SUNSPOTS BY UMBRAL MERGING
}

\author{
HAROLD ZIRIN
}

Big Bear Solar Observatory, California Institute of Technology, Pasadena, CA 91125, U.S.A.

(Received 24 September, 1986; in revised form 25 August, 1987)

\begin{abstract}
The formation of a sunspot during the emergence of a new group is described. The spot forms from a cluster of small umbrae that do not converge. Rather, the individual umbrae enlarge and merge into a spot covering the same area. The formation of each umbra is accompanied by an intensification of the arch filament anchored in it. The formation of the sunspot produces no apparent change in the total field.
\end{abstract}

\section{Introduction}

The emergence and formation of sunspots in an emerging flux region (EFR) is a remarkable process. In a short time a structure that may last for weeks is formed, and the geometry of the EFR process (Weart, 1970; Chou, 1986) gives clear evidence of emergence of flux loops. How these loops give rise to large spots is a complex problem involving the combination of magnetic buoyancy and the cooling process. Several authors (Vrabec, 1974; McIntosh, 1981; Zwaan, 1985) have demonstrated the merging of larger spots from different EFR's to form a large spot, but the formation of the individual spots has not been hitherto revealed. We present here a particularly fine example of the formation of a $p$-spot in which the spot formation is continuously observed in all parts of the $\mathrm{H} \alpha$ line as well as the $\mathrm{Ca} \mathrm{K}$-line and magnetograms. Contrary to the familiar 'oak tree' model in which spots are supposed to converge to form a large spot, a group of tiny umbrae grow and merge to form a mature spot without any decrease in overall area.

These data also furnish an opportunity to observe the connection of prominent arch filaments with the formation of umbrae. We have long suspected this to occur as a byproduct of spot formation.

\section{Observations}

The region BBSO No. 0116 was observed on $12 \mathrm{July,} 1983$ for a period of about 10 hours at Big Bear. Observations were less frequent on the 13th. Figures 1 and 2 present observations of the EFR during this period in different points in the $\mathrm{H} \alpha$ line. During the period of observation the Zeiss birefringent filter was continually tuned back and forth from $-2 \AA$ to $+2 \AA$ across the line under computer control. On the left side of Figure 1 we present a series of frames in the blue wing of $H \alpha(-0.8 \AA$ in (a) and (b) and $-2 \AA$ in 1(c) compared with frames at $+0.8 \AA$ in (d) and $\mathrm{H} \alpha$ centerline (e) and (f)) at right. These show the well-known difference in appearance of arch filament 


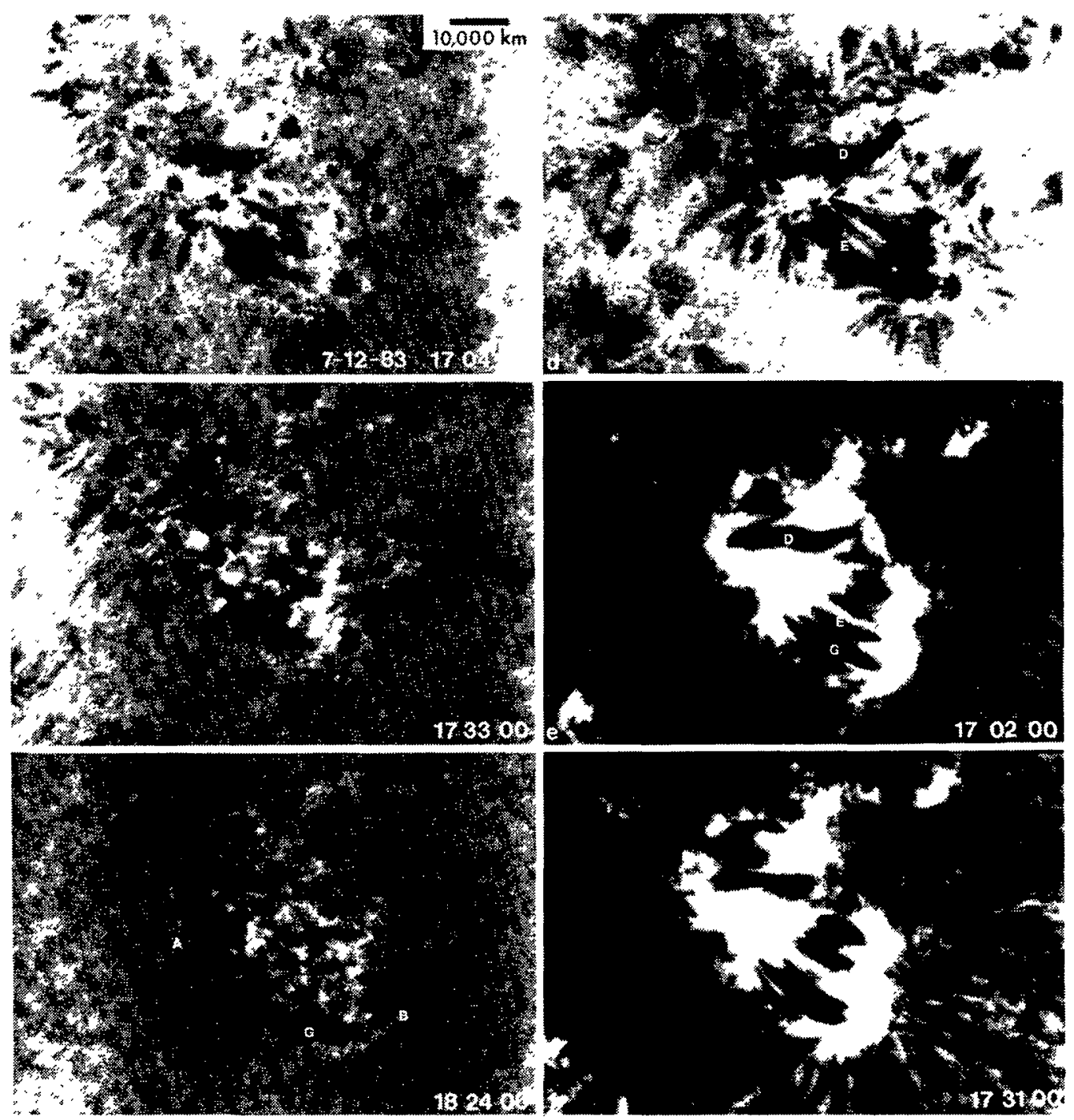

Fig. 1. Early emergence of the regions with the scanning $\mathrm{H} \alpha$ filter: left, blue wing, showing upward moving loop tops; (d), red wing showing downflow of arch material into the umbrae; (e) and (f): centerline showing AFS and bright plage. In all images $S$ top, $W$ left.

systems (AFS) first noted by Bruzek (1967). The tops of the AFS loops are visible in the blue as the magnetic arch rises toward us and the bases are visible in the red as the material pours down. In Figure 1(d) we clearly see the material pouring into the spots. The blue wing frames show the spots clearly because the AFS is strongly red shifted. The downward moving material at the arch bases is much more prominent than the blue-shifted arch tops; in a steady state they should be equal. This means that material either piles up at the bottom or cools as it falls.

A number of Ellerman bombs are visible in the line wings as is typical in such emerging flux regions; we can find nothing systematic in their location or occurrence. The growing region has two major arch filament systems, marked $D$ and $E$ in Figure 1(d). These connect the $p$-polarity spots $F$ and $A$ with the follower spots $C$ and $B$, respectively. $D$ is a somewhat twisted group of arches connecting the compact spots $C$ and $F$; $E$, on 

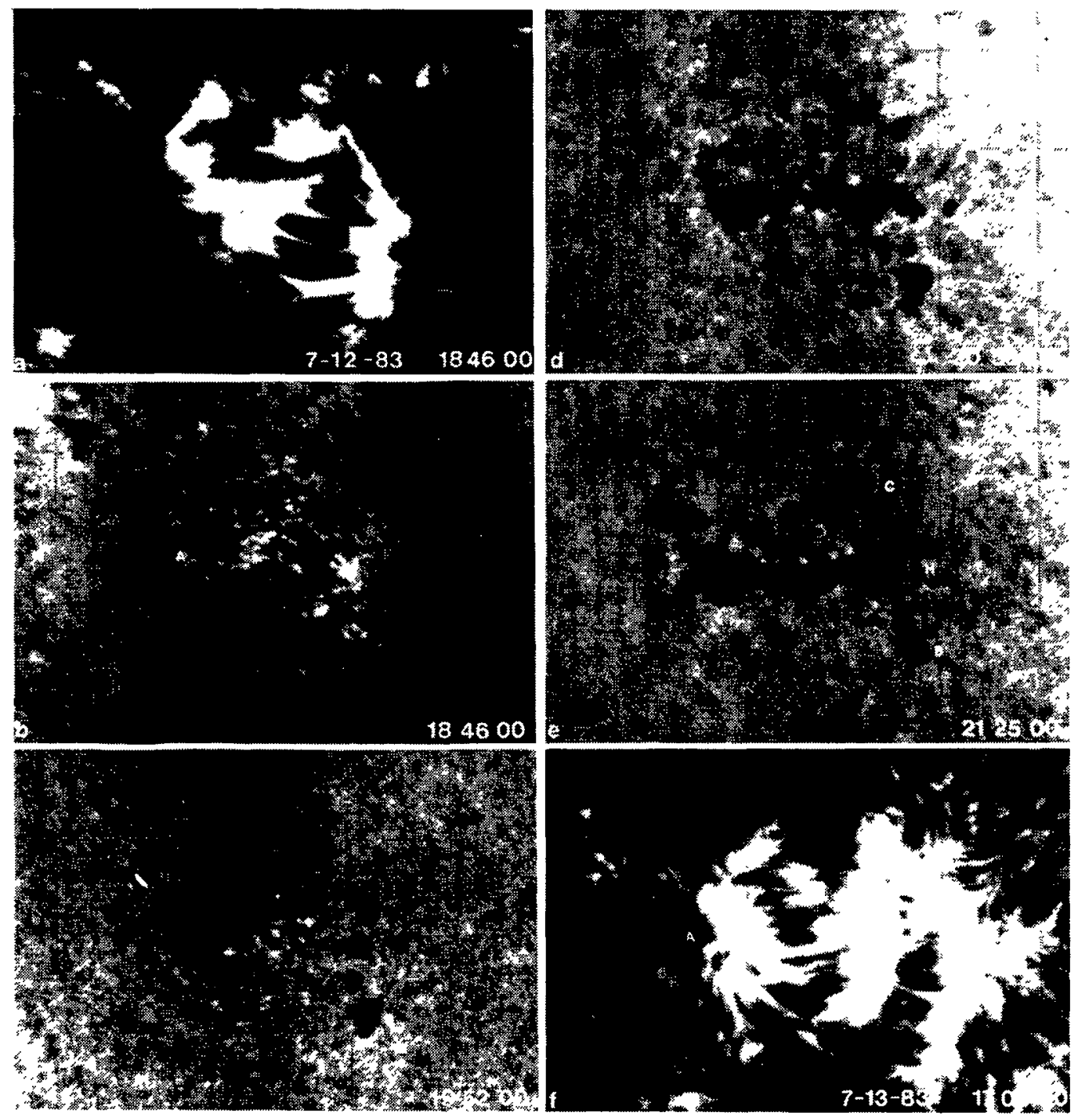

Fig. 2. Later development; the left frames are blue wing or line center, the right frames, red wing or line center. The merging into the $p$ spot takes only a few hours, but the penumbra developed overnight and appears in the last frame.

the other hand, is an arcade of parallel arches connecting the series of small spots near $A$ with various small spots in the neighborhood of follower spot $B$. In Figure 2 we see the further development of the region; Figure 2(a) shows that the concentrated arch filament $D$ connected with the formation of follower spot $C$ has been replaced by a series of individual arch filaments connected to a number of new umbrae. This is an example of the close association between the arch intensity and umbra growth; each time we find a new umbra or spot growing we find a large dark arch such as $D$ or $G$ between it and the opposite pole. This may be associated with the cooling process.

The main result of these observations is the development pattern of the sunspot $A$. It first appears in Figure 1(a) as two parallel rows of small spots, about eight in all. These are seen in Figure 1(d) to be at the footpoints of the arch filaments coming from the following spots around $B$. In the center of $\mathrm{H} \alpha$ the spots are almost invisible because of 
the bright overlying material. In Figure 1(b) we see that the leading members of this group have already merged, and in Figure (1c) we see that only 80 min later they have almost completely merged into an $S$-shaped spot. But the overall size is no greater than Figure 1(a). Spot formation in this case does not necessarily appear to be convergence of spots but filling in of the space between the spots. In Figure 1(b) this process is seen to have continued further and in Figure 2(c) the $S$-shaped spot is fully developed. In Figure 1(d), about 4 hours after the beginning of our observations, the $S$-shaped spot has grown into two parts which now have a somewhat different magnetic connection,
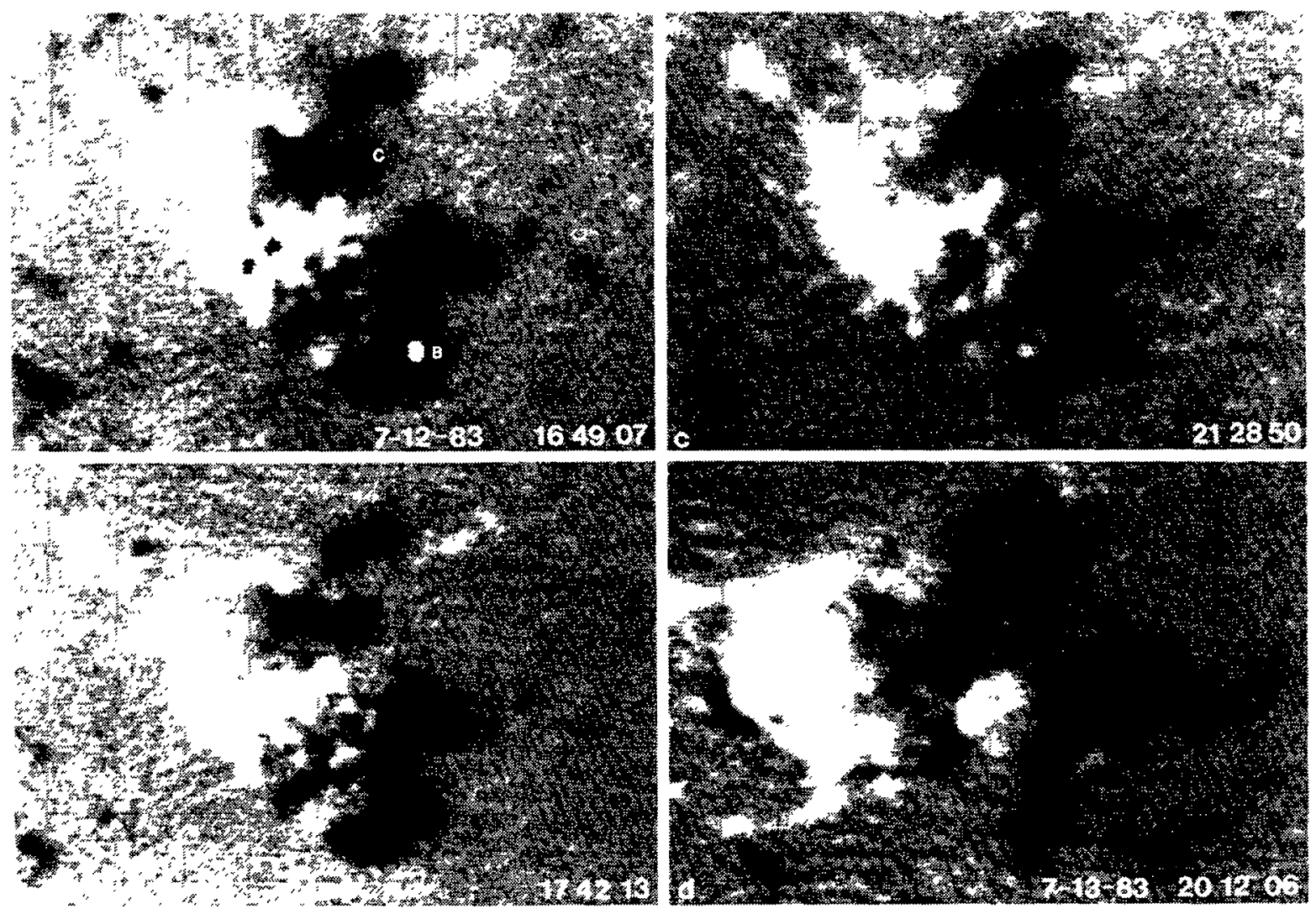

Fig. 3. Four videomagnetograms of the region development. Despite the substantial changes in the spot on the first day, the only apparent change in the magnetic fields is separation. In the last frame, taken the next day, new flux loops have emerged.

being joined to a spot which we have marked $H$ by a long arch filament. During this period of the rapid growth of spot $A$, the other sunspots in the group changed very little. If we examine Figure 3, which shows magnetograms of the region on both the 12th and 13 th, we see that there is little change in the longitudinal field except for the general westward (left) motion of the white $(p)$ polarity. This is consonant with the result of Wallenhorst and Topka (1982); the appearance or disappearance of the spot appears to be a matter of change in cooling rather than flux change. While the magnetograph was only calibrated once during the day, the surrounding quiet-Sun magnetic elements 
show little change, and it would be surprising if the sensitivity were changing just sufficient to offset some growth in the field.

Our telescope in Tel Aviv was out of operation on the 13th. At the behest of the referee of Solar Physics we sought observations from elsewhere of the overnight development of the region, and Prof. Dezsö kindly furnished us a number of white light observations from Debrecen. These show the consolidated umbra with no penumbra from 05:50 UT on July 13 to $11: 19 \mathrm{UT}$, but substantial change in the structure of the umbra, confirmed by 'equidensitograms' obtained by Dr O. Gerlei. So the main spot was still in the process of forming. The first Debrecen frames show hints of the $S$-shaped structure remaining in the umbra. The referee of Solar Physics pointed out that there is some rotation in the spot on July 12 (Figures 1(c)-(d). I believe this is only apparent, the result of growth of the leading part of the spot. The Debrecen images show a $90^{\circ}$ rotating of the long axis of the spot in $6 \mathrm{hr}$; here, too, I fear this may be an artifact of growth.

Our last picture in Figures 2 and 3 shows the region on the next day, with a mature spot $A$ with a penumbra. Spot $F$ is barely visible in this $\mathrm{H} \alpha$ frame, and spot $B$, which disappeared on the 13th, is no longer visible. The arch filament system remaining in Figure 2(f), was now double as a result of new $p$ flux (Figure 3(d) arising in the trailing fields. One would expect three sets of arches in this case but the white flux appears unconnected to the dark flux ahead of it. Comparison of Figures 3(c) and (d) shows that both white (positive) and dark flux came up overnight in the center of the region. The flux loops connecting the main $p$ and $f$ polarities are high above the surface now and no longer visible in $\mathrm{H} \alpha$. The white polarity in the following area disappeared by 23:00 UT, but we did not observe continuously in this period. The regular radial form of the penumbra supports the view that there was no rotation.

\section{Discussion}

The initial process of formation of spot $A$ took no more than 6 or 7 hours, but the formation of the penumbra required 24 hours or more. The scale in Figure 1(a) shows the spot diameter to be about $10000 \mathrm{~km}$. During this period there was only limited growth in the total $p$ flux. The separation of $p$ and $f$ polarities during the entire span of Figure 3 took place at a steady $0.3 \mathrm{~km} \mathrm{~s}^{-1}$. Comparison with nearby magnetic features shows the motion to be entirely in the $p$ spot.

One interpretation of the images of spot $A$ is geometric: they represent the successive intersections of its field with the surface plane. In that case we may reconstruct a three-dimensional picture of the field. If this is correct, the loops above the surface, although probably considerably expanded in cross-section compared to the surface, are clumped in the $S$-shaped configuration. On the other hand it is possible that the evolution from small spots to $S$-shape to flat field is a physical development. In that case the growing spot fields produce a thermal instability which cools the plasma and produces the spots. The cooling of spots has been discussed by Chou (1986), who found it to take place over a period similar to that we see here. Thus the field is fully emerged in Figure 1(a), but the cooling process only produced a few umbrae. The appearance 
of dark arch filaments during the umbra formation suggests the physical rather than geometric view.

The magnetograms also contradict the popular 'oak tree' model of flux convergence. In that picture (which is illustrated by Zwaan, 1985, Figure 1), the top of the flux loop is expanded, so that the decrease of the area of intersection of flux ropes and photosphere as the loops rises produces an apparent convergence of small flux elements from the neutral line toward the main poles. That this occurs with larger spots is not in doubt; good examples are given by McIntosh (1981) and others. But those cases apparently involve separate, larger flux loops. In our data we are looking at an individual EFR and the formation of a single, modest-sized sunspot, which at least in this case formed as shown. It is difficult to reconcile the constant emergence of AFS with the apparent absence of a flow of small magnetic elements to the main poles. We examined the magnetograms, which were obtained every minute, and found no evidence of such behavior. In all cases a single set of loops with two poles emerges and, as we see in Figure 3, spreads apart without much change. All of our $\mathrm{H} \alpha$ and $\mathrm{K}$-line images confirm this single-evolution picture. Sunspots then form this loop, which does not increase in total flux. The additional flux between the poles on the 13th did not spread outward to enhance the poles, but remained as plage. Larger sunspots or complexes cannot grow that way and require the convergence of larger, distinct EFR's.

In our understanding of individual spot formation we must, therefore, confront the problem of an equal overall flux appearing as plage or sunspot of different darkness and extent. This may be related to the fact that some spots are short lived, while naked sunspots may last several rotations (Liggett and Zirin, 1983).

\section{Acknowledgements}

This research was supported by the NSF Atmospheric Sciences Section under ATM 8513577 and NASA under NGL 05002 034. I am indebted to Randy Fear, Dean Chou and Bill Marquette for their assistance in obtaining the observations.

\section{References}

Bruzek, A.: 1967, Solar Phys. $2,451$.

Chou, D.-Y.: 1986, Ph.D. Thesis, Caltech.

Liggett, M. A. and Zirin, H.: 1983, Solar Phys. 84, 3.

McIntosh, P. S.: 1981, in Cram and Thomas (eds.), The Physics of Sunspots, Sacramento Peak Obs., Sunspot.

Vrabec, D.: 1974, in R. G. Athay (ed.), 'Chromospheric Fine Structure', IAU Symp. 56, 201.

Wallenhorst, S. G. and Topka, K. P.: 1982, Solar Phys. 81, 33.

Weart, S. R.: 1970, Astrophys. J. 162, 987.

Zwaan, C.: 1985, Solar Phys. 100, 397. 